

\title{
Epitaxial Growth Simulation Employing a Combined Molecular Dynamics and Monte Carlo Approach
}

\author{
C. H. Grein \\ Physics Department \\ University of Illinois at Chicago \\ Chicago, IL 60607-7059 \\ Roy Benedek \\ Materials Science Division \\ Argonne National Laboratory \\ Argonne, IL 60439 \\ and \\ T. de la Rubia \\ Chemistry and Materials Science Division \\ Lawrence Livermore National Laboratory \\ Livermore, CA 94550
}

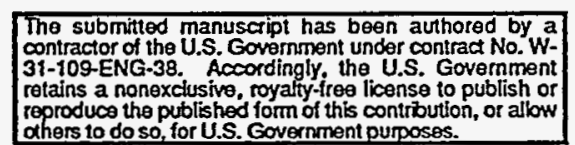

July, 1995

$/ \mathrm{sm}$

Submitted for the Proceedings of Virtual MBE Workshop on MBE Growth Modeling, Malibu, CA, June 15-17, 1995

This work is supported by the Division of Materials Sciences, Office of Basic Energy Sciences of DOE, under contract No. W-31-109-ENG-38. 


\title{
Epitaxial Growth Simulation Employing a Combined Molecular Dynamics and Monte Carlo Approach
}

\author{
C.H. Grein \\ Physics Department, University of Illinois at Chicago, Chicago, IL 60607-7059 \\ R. Benedek \\ Materials Science Division, Argonne National Laboratory, Argonne, IL 60439 \\ T. de la Rubia \\ Chemistry and Materials Science Division, \\ Lawrence Livermore National Laboratory, Livermore, CA 94550
}

\begin{abstract}
The epitaxial growth of $\mathrm{Ge}$ on $\mathrm{Si}(001)$ is simulated by employing a hybrid approach based on molecular dynamics to describe the initial kinetic behavior of deposited adatoms and Monte Carlo displacements to account for subsequent equilibration. This method is well suited to describe initial nucleation and growth. Stillinger-Weber potentials are employed to describe interatomic interactions.
\end{abstract}




\section{Introduction}

The rapid development of sophisticated epitaxial growth techniques for semiconductors has prompted the desire to develop modeling methods that are capable of helping in the interpretation of experimental data and to potentially aid in the selection of optimum growth conditions. Methods ranging from ab-initio calculations, which are useful for making reliable predictions regarding specific questions pertaining to atomic-scale volumes of the actual sample, to continuum approaches, which address general non-material specific properties of growth, are under extensive scrutiny. Intermediate between these two approaches are the molecular dynamics (MD) and Monte Carlo (MC) methods. The MD method is computationally practical for describing growth phenomena on ps to $\mu$ s time scales, whereas the MC method is practical for $\mu$ s to s time scales. We discuss here a hybrid $\mathrm{MD} / \mathrm{MC}$ approach designed to combine. the advantages of the MD and MC. It permits the study of epitaxial growth over time scales longer than either approach alone allows.

\section{Methodology}

To simulate MBE epitaxial growth at the relatively slow deposition rates employed experimentally, the arrival of individual atoms or clusters at the substrate must be treated as essentially isolated events. The physics of each of these events is governed by at several distinct time scales. The collision with the substrate occurs in a time of the order $10^{-13} \mathrm{~s}$, during which the particle is either reflected or sticks to the surface. If sticking occurs, local relaxation and dissipation of the kinetic energy of the particle occurs on a scale of $10^{-12} \mathrm{~s}$. Molecular reactions and hopping occur on time scales of $10^{-12}$ to $10^{-6} \mathrm{~s}$. Since the next particle to arrive at the substrate in the vicinity of the first one occurs several orders of magnitude later in time, the already deposited particles undergo thermally activated diffusion, reevaporation and other rearrangements in the interim.

The most common atomistic methods of $\mathrm{MBE}$ simulation are based on either Monte Carlo (MC) or MD algorithms, each of which has adrantages and disadvantages. In principle, MD is superior because it can describe dynamic, nonequilibrium 
systems such as epitaxially growing layers, but it is typically restricted to integrated time intervals of tens of picoseconds $[1,2]$. On the other hand, $M C$ (we restrict our attention to those methods based on importance sampling) cannot incorporate time dependence because its algorithm depends on thermodynamic equilibrium[3, 4]. MD is capable of simulating the collision and short-term relaxation phases described above, but not the rearrangements during the hiatus between successive particle arrivals. The latter, however, can be approximately simulated with MC methods. Both methods require accurate representations of atomic interactions.

We therefore discuss here a hybrid approach, in which MD is used to model the short term kinetic behavior following the arrival of each new particle at the substrate; and $\mathrm{MC}$ models the subsequent local equilibration between deposition events. In principle, of course, a unified approach to the entire process would be more desirable than the hybrid approach adopted here. Unfortunately, in view of the disparate time scales that characterize the deposition and growth process, the hybrid approach appears to be the best alternative available.

Molecular dynamics is a widely practiced simulation technique[5]. It has been applied to study the crystal growth by atom or cluster deposition of various materials [2,613]. Likewise, many purely Monte Carlo growth simulations have been performed[1427]. These works, however, have been applied primarily to considerably simpler systems than the heteroepitaxial layered structures that are the subject of present day experimental research.

The complex interactions between adatoms and substrate atoms of a different atomic species require continuous-space simulations. Lattice gas models such as the solid-on-solid (SOS) model, while appropriate for homoepitaxy[25], cannot describe lattice-mismatch associated defects in heteroepitaxial systems, surface reconstructions, or dynamics associated with phonons[14]. Moreover, the formation of complex interface compounds, while possible in $\mathrm{MD} / \mathrm{MC}$ simulations, would be prohibited with a SOS model.

The adopted MD methodology is similar to that employed by Ethier and Lewis[2] to model $\mathrm{Si}_{1-x} \mathrm{Ge}_{x}$ growth on $(100) \mathrm{Si}(2 \times 1)$. It incorporates the sequential deposition of energetic atoms onto a substrate, with damped layers below the substrate surface to simulate the thermalization of the deposited species. Periodic boundary conditions 
are applied in the plane of the substrate. Typically[2], MD simulations of the crystal growth of covalent systems, even on the Cray X-MP, have been limited to cells with substrate areas of a few tens of atoms and only a few hundred atoms in the entire simulation cell. The treatment of larger unit cells would be a considerable advantage. A larger substrate area, for example, would allow a greater variety of surface reconstructions, orientations, and miscuts, as well as structures of the deposited species. The performance of MD on massively parallel computers makes the treatment of large cells feasible. MD readily lends itself to parallel processing, because the elements of the force vector may all be calculated independently. Results presented here are obtained employing a sequential MD methodology, but work is underway to implement a parallel version.

After the MD simulation of the adsorption of a single atom or cluster, the system is equilibrated with the dynamic MC method described in ref. [22]. Specifically, several hundred $M C$ steps are repeated on all atoms by shifting their positions by the displacement vectors with components

$$
\delta_{\nu}=\frac{2 k_{B} T}{F_{\nu}} \ln \left[\frac{1}{\alpha}+\left(\alpha-\frac{1}{\alpha}\right) R\right]
$$

where $\alpha=\exp \left(\left|\epsilon F_{\nu} / 2 k_{B} T\right|\right), F_{\nu}$ is the $\nu$ th component of the force on the atom under consideration, $T$ is the temperature, $\epsilon$ is the maximum length of an atomic displacement, and $R$ is a random number in the range $[0,1]$. Then the next deposition event is permitted to occur, and is treated employing the same MD/MC approach.

The accuracy of the interatomic potentials employed in a growth simulation is crucial. The potentials should be capable of describing interatomic interactions under the wide variety of configurations that occur during growth. Classical potentials that have been developed employing a large database including numerous crystal structures involving different bond angles are desirable. Extensive testing of the potentials should establish that they predict observed surface reconstructions and are in agreement with ab-initio results for total energies and phonon frequencies. We choose the extensively tested Stillinger Weber potentials[28] for describing interatomic interactions during the growth of $\mathrm{Ge}$ on $\mathrm{Si}$. Stillinger and Weber's original choice of $\mathrm{Si}$ parameters is employed for bulk $\mathrm{Si}$, and another parameterization of the Stillinger Weber potential for bulk Ge[29]. Si-Ge interaction parameters are interpolated from 
those of pure $\mathrm{Si}$ and pure Ge employing the methods in ref. [2].

\section{Results}

The Si substrate in the supercell employed to model epitaxial growth consists of 8 atomic layers each containing 50 atoms. Periodic boundary conditions are applied in the [100] and [010] directions. The lowest layer of atoms is fixed at absolute zero temperature, the 6 layers above it are maintained at $\mathrm{T}=500^{\circ} \mathrm{C}$, and: the uppermost layer is free from any thermal constraints. The substrate temperature is maintained by rescaling the velocities of the central 6 layers every $5 \mathrm{fs}$. A $2 \times 1$ reconstruction of the $\mathrm{Si}(001)$ surface is observed to take place within $100 \mathrm{fs}$ of the start of the simulation, but dereconstruction takes place during the deposition of the first monolayer of $\mathrm{Ge}$. The deposited Ge atoms all have an initial kinetic energy of $0.21 \mathrm{eV}$, and are directed . vertically towards the Si surface from a randomly chosen location in a plane $11 \AA$ above the surface. The first Ge atom is projected 200 fs after the start of the simulation, and MD time steps of $1 \mathrm{fs}$ each are continued until $200 \mathrm{fs}$ after this atom first interacts with the surface. This is comparable to the time scale between depositions employed in earlier purely-MD simulations[1, 2]. However, we follow this by $200 \mathrm{MC}$ steps; which are performed on an all atoms. This is followed by the projection of the next Ge atom and the above $\mathrm{MD} / \mathrm{MC}$ procedure is repeated. In all, 4 atomic layers of $\mathrm{Ge}$ are deposited. We plan to perform future simulations on parallel processors, which will permit the consideration of more MD/MC steps between depositions.

Figure 1 plots the atomic positions of the Ge and Si atoms every 160 and 1000 MD time steps respectively. Displacements during the $\mathrm{MC}$ stages of the simulation are not plotted. Each point represents the atomic position projected onto a plane perpendicular to [100]. A significant thermal gradient is visible in the substrate. A clearer view of the epilayers is provided by Fig. 2. Plotted are the atomic positions in the upper layers at the end of the simulation. Ge atoms are found to interdiffuse one atomic layers into the $\mathrm{Si}$ substrate, with the $\mathrm{Si}$ atoms that are knocked out incorporating in the epilayers. Two dimensional epitaxial growth of the Ge epilayers is observed over approximately three monolayers, after which the strain associated with the $4 \%$ lattice mismatch between $\mathrm{Si}$ and Ge induces three-dimensional growth. This 
is in agreement with an earlier MD simulation[2] and experimental observations[30].

In conclusion, the combination of $\mathrm{MC}$ with $\mathrm{MD}$ extends the time scale of epitaxial growth simulations over those that employ a purely MD methodology. This permits a more realistic description of the growth dynamics between atomic depositions. Results presented here indicate that the hybrid approach is capable of reproducing earlier purely MD results which required considerably greater computational effort. Moreover, the simulations produce results in good agreement with experimental data.

\section{Acknowledgements}

We are grateful to E. Kaxiras for helpful discussions. R.B. was supported at Argonne National Laboratory by the U.S. Department of Energy under Contract No. W-31-109-ENG-38. T.D.D. was supported at Lawrence Livermore National Laboratory by the U.S. Department of Energy under contract no. W-7405-ENG-48. 


\section{References}

[1] M. Schneider, I.K. Schuller, and A. Rahman, Phys. Rev. B 36 (1987) 1340.

[2] S. Ethier and L.J. Lewis, J. Mater. Res. 7 (1992) 2817.

[3] N. Metropolis, A.W. Rosenbluth, N.M. Rosenbluth, A.H. Teller, and E. Teller, J. Chem. Phys. 21 (1953) 1087.

[4] A. Madhukar and S.V. Ghaisas, CRC Crit. Rev. Solid State Mater. Sci. 14 (1988) 1.

[5] M. P. Allen and D. J. Tildesley, Computer Simulation of Liquids (Clarendon Press, Oxford, 1987).

[6] M. Kitabatake, M. Deguchi, and T. Hirao, J. Appl. Phys. 74 (1993) 4438.

[7] C. Roland and G.H. Gilmer, Phys. Rev. B 47 (1993) 16286.

[8] M.F. Crowley, D. Srivastava, and B.J. Garrison, Surf. Sci. 284 (1993) 91.

[9] A. Miyamoto, K. Takeichi, T. Hattori, M. Kubo, and T. Inui, Jap. J. Appl. Phys. Part 131 (1992) 4463.

[10] U. Landman and W.D. Luedtke, Appl. Surf. Sci 60 (1992) 1.

[11] C.M. Gilmore and J.A. Sprague, J. Vacuum Sci Tech. A 10 (1992) 1597.

[12] H.P. Kaukonen and R.M. Nieminen, Phys. Rev. Lett. 68 (1992) 620.

[13] T.J. Moran, I.K. Schuller, and R. Ramirez, Phys. Rev. B 49 (1994) 5729.

[14] B.W. Dodson and P.A. Taylor, Phys. Rev. B 34 (1986) 2112.

[15] W.M. Plotz, K. Hingerl, and H. Sitter, J. Cryst. Growth 115 (1991) 186.

[16] J.M. McCoy and P.A. Maksym, Semicond. Sci. Technol. 6 (1991) 141.

[17] H.J. Osten, Thin Solid Films 215 (1992) 14.

[18] N. Georgiev, A. Milchev, M. Paunov, Surf. Sci. 264 (1992) 455.

[19] B.A. Joyce, T. Shitara, A. Yoshinaga, D.D. Vvedensky, J.H. Neave, and J. Zhang, . Appl. Surf. Sci. 60 (1992) 200.

[20] M.D. Rouhani, A.M. Gue, M. Sahlaoui, and D. Estsve, Surf. Sci. 276 (1992) 109.

[21] I.K. Marmorkos and S. Das Sarma, Phys. Rev. B 45 (1992) 11262.

[22] G. Dereli, Molec. Simul. 8 (1992) 351.

[23] J. Kew, M.R. Wilby, and D.Vvedensky, J. Cryst. Growth 127 (1993) 508. 
[24] P.W. Rooney and F. Hellman, Phys. Rev. B 48 (1993) 3079.

[25] T. Kawamura, Prog. Surf. Sci. 44 (1993) 67.

[26] J. Xing and H.L. Scott, Phys. Rev. B 48 (1993) 4806.

[27] M.I. Larsson and G.V. Hansson, Surf. Sci. 292 (1993) 98.

[28] F.H. Stillinger and T.A. Weber, Phys. Rev. B 31 (1985) 5262.

[29] K. Ding and H.C Andersen, Phys. Rev. B 34 (1986) 6987.

[30] Y. Kataoka, H. Ueba, and C. Tatsuyama, J. Appl. Phys. 63 (1988) 749. 


\section{Figure Captions}

Figure 1. Epitaxial growth simulation of $\mathrm{Ge}$ on $\mathrm{Si}(001)$. Plotted are the positions of the $\mathrm{Si}$ and $\mathrm{Ge}$ atoms projected onto a plane perpendicular to the [100] direction every 1000 and $160 \mathrm{MD}$ time steps, respectively. The horizontal dashed line marks the interface between the original Si surface and the Ge epilayers. The Si substrate is below the dashed line, and the Ge epilayers above.

Figure 2. Atomic positions in the supercell after the simulation, plotted by atomic layer. Layer 8 is the Si surface before Ge growth starts. The filled circles represent $\mathrm{Si}$ atoms, and the empty ones Ge atoms. 


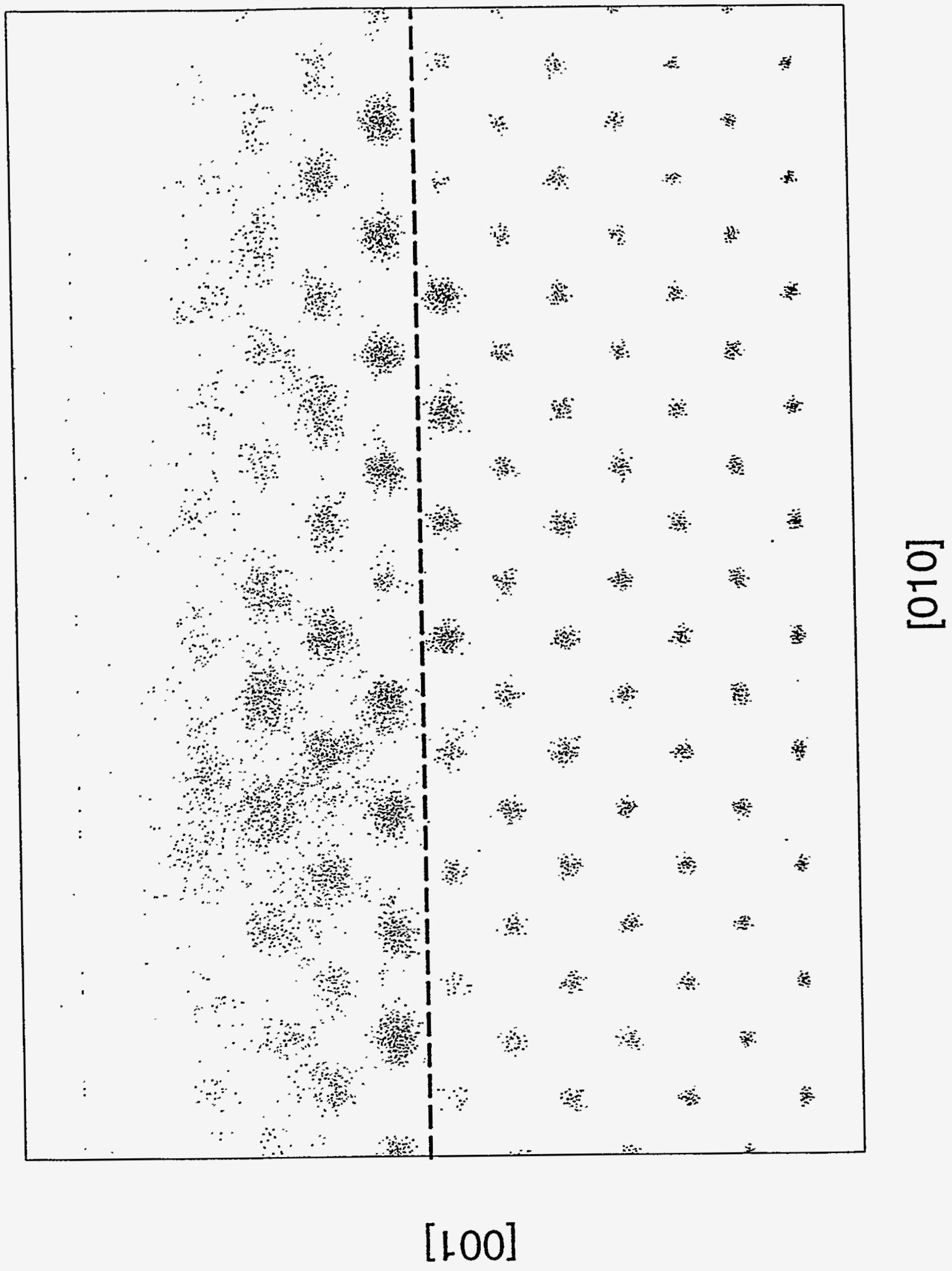



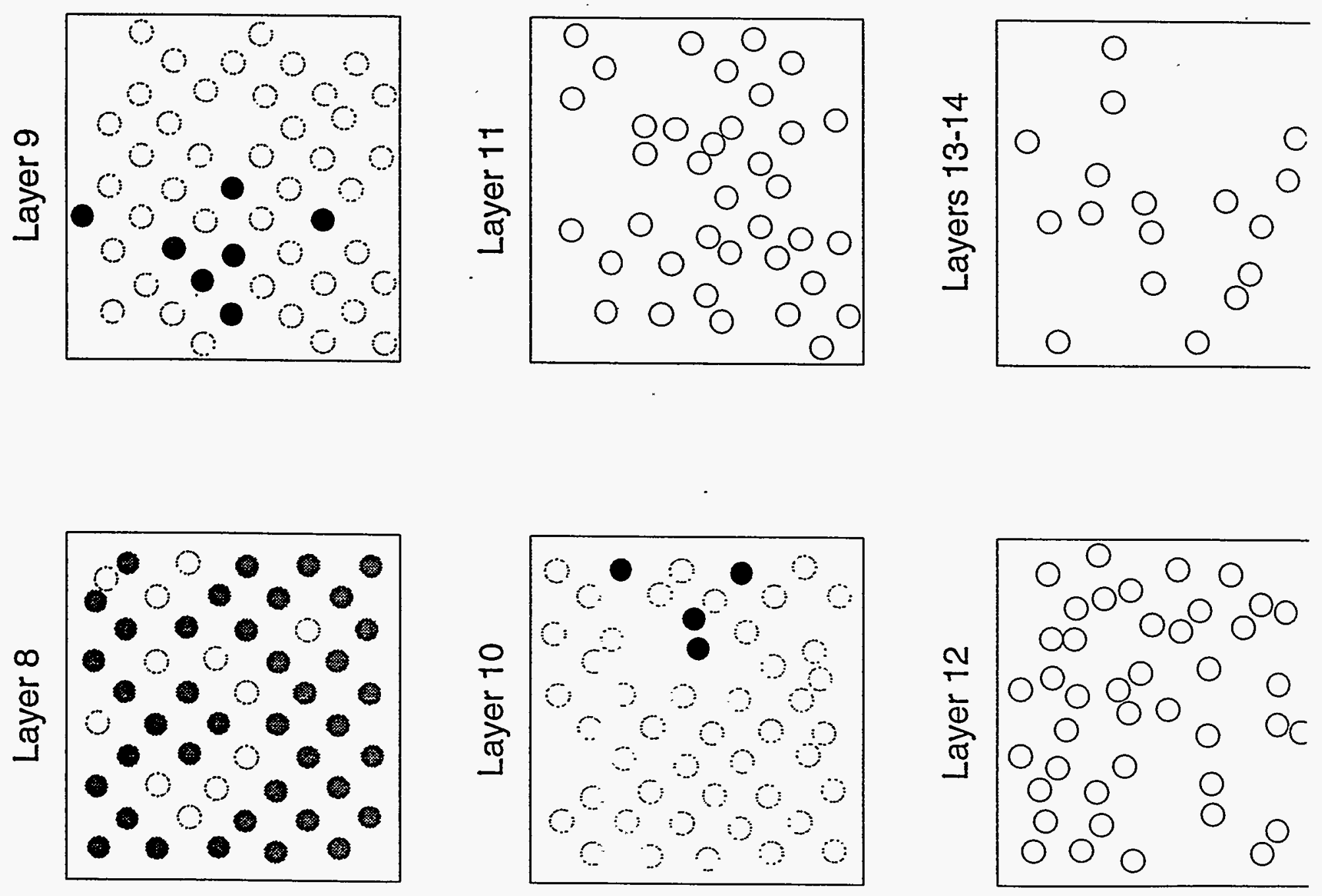